\title{
Uso Racional de Medicamentos: Relato de Experiência no Ensino Médico da Unesc, Criciúma/SC
}

\author{
Rational Use of Medicines: Report of an \\ Experience in Medical Education at Unesc \\ Criciúma, Santa Catarina State, Brazil
}

Iane Franceschet-de-Sousa ${ }^{I}$ Andressa Biscaro ${ }^{I I}$

Fernanda Biscaro ${ }^{I I I}$

Marcelo Soares Fernandes ${ }^{I V}$

\section{PALAVRAS-CHAVE}

- Medicina Baseada em Evidências.

- Educação Médica.

- Uso de Medicamentos.

\section{KEYWORDS}

- Evidence-Based Medicine.

- Education, Medical

- Drug Utilization.

Recebido em: 09/01/2009

Reencaminhado em: 01/08/2009

Reencaminhado em: 27/10/2009

Aprovado em: 06/11/2009

REVISTA BRASILEIRA DE EDUCAÇÃo MÉDICA
${ }^{\text {I}}$ Faculdade Estácio de Sá, Campo Grande, MS, Brasil.

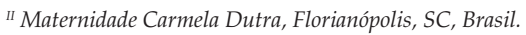

${ }^{I I I}$ Universidade Federal de Santa Catarina, Florianópolis, SC, Brasil.

${ }^{I V}$ Universidade do Extremo Sul Catarinense, Criciúma, SC, Brasil.
RESUMO

O presente estudo objetivou disseminar entre os alunos do curso de Medicina da Unesc a lógica do Uso Racional de Medicamentos e suas aplicações na prática médica. Utilizou-se como modelo o curso de formação sobre Uso Racional de Medicamentos promovido pela OMS, por meio do método didático-pedagógico da aprendizagem baseada em problemas, a mesma concepção utilizada pelo curso de Medicina da Unesc. Com apoio e financiamento da Diretoria de Pesquisa e da Diretoria de Extensão e Ação Comunitária (Unesc), o trabalho foi desenvolvido a partir de duas vertentes: a primeira visou capacitar acadêmicos do curso de Medicina na lógica do Uso Racional de Medicamentos; a segunda vertente objetivou desenvolver ações educativas na comunidade dos bairros adjacentes à universidade, envolvendo temas como Uso Racional de Medicamentos. Foram capacitados 35 alunos e realizadas intervenções na comunidade que abrangeram 689 pessoas. Os acadêmicos envolvidos neste trabalho passaram a visualizar a prescrição de medicamentos de forma mais racional e ainda adquiriram a noção da importância de priorizar uma lista de medicamentos essenciais, tendo como base condutas pautadas nas melhores evidências disponíveis.

The current study aimed to disseminate the logic of rational use of medicines and its application in medical practice among medical students at UNESC, Santa Catarina State, Brazil. The study's model was the training course on Rational Use of Medicines promoted by the World Health Organization (WHO), using the problem-based teaching-learning method, a strategy adopted by the UNESC School of Medicine in general. With support and funding from the Office of the Dean for Research and the Office of the Dean for Extension and Community Action (UNESC), the project was developed along two lines: first, training undergraduate medical students in the logic of Rational Use of Medicines; second, develop educational activities in the community, in neighborhoods near the university, on themes related to Rational Use of Medicines. Thirty-five students were trained, and community interventions were conducted with a total of 689 community members. The medical students involved in this project began to view prescriptions more rationally and gained a notion of the importance of prioritizing a list of essential drugs, for treatment supported by the best available evidence. 


\section{INTRODUÇÃO}

Na prática médica quotidiana, defronta-se o profissional com a difícil tarefa de escolher métodos diagnósticos e tratamentos eficazes. Tradicionalmente, tais tomadas de decisão têm-se baseado em princípios fisiopatogênicos, raciocínio lógico, observação pessoal e intuição que, em conjunto, constituem a chamada experiência do clínico. Isso torna a intervenção muito subjetiva e de difícil extrapolaçãoํ.

Na década de 1990, surgiu um novo modelo de pensamento - a Medicina Baseada em Evidências - , que reforça a experiência clínica por meio da aplicação da melhor informação científica disponível, valorizando o paciente quanto a suas peculiaridades e expectativas e objetivando atendimento mais correto, ético e cientificamente embasado. Neste paradigma, pesquisa e prática clínica não mais se dissociam e fazem parte de um processo sistemático e contínuo de autoaprendizado e autoavaliação, sem o que as condutas se tornam rapidamente desatualizadas e não racionais ${ }^{1,2}$.

A Conduta Baseada em Evidências ainda é desconhecida por grande parte dos prescritores. Os profissionais e acadêmicos da área da saúde, sobretudo da área médica e farmacêutica, devem conhecer os princípios que regem esta prática para otimizar os benefícios e minimizar os riscos na utilização de medicamentos pela população.

A partir desta concepção, o presente trabalho visou disseminar entre os acadêmicos do curso de Medicina da Universidade do Extremo Sul Catarinense (Unesc) a lógica do Uso Racional de Medicamentos e suas aplicações na prática médica.

\section{Promoção do Uso Racional de Medicamentos: um Desafio Contínuo}

Uma questão inicial na discussão do estabelecimento dos diferentes tipos de intervenções para promoção ou avaliação do uso racional consiste na identificação dos possíveis problemas derivados do uso não racional, como o sobreúso de medicamentos (por exemplo, a prescrição excessiva de injetáveis) e o uso incorreto (como o de antiinflamatórios em situações não inflamatórias). Observa-se que o uso inapropriado de medicamentos pode ter consequências como surgimento de eventos adversos (incluindo os letais); de resistência a antibacterianos; farmacodependência; entre outros ${ }^{3}$.

Além disso, o uso inadequado e excessivo de medicamentos acarreta um desperdício de recursos, com frequência pagos pelos pacientes, e trazem como consequência um considerável prejuízo ao paciente devido à falta de resultados positivos e à incidência de reações adversas. Já as doses inadequadas têm como consequência um alto índice de morbimortalidade, sobretudo a raiz de infecções infantis e doenças crônicas, como hipertensão, diabete, epilepsia ou doenças mentais ${ }^{4}$. Em situações onde há grande número de atendimentos, como na atenção primária à saúde, deve-se ter o cuidado de não utilizar a prescrição como forma mais simples de encerrar uma consulta ${ }^{5}$.

A integração entre prescritores e dispensadores permite, mediante a combinação de conhecimentos especializados e complementares, o alcance de resultados eficientes, beneficiando o paciente ${ }^{6}$. No que tange ao uso de medicamentos, tanto na prescrição quanto na dispensação, existem forças contrárias que impulsionam a utilização irracional, como a influência da indústria farmacêutica e da mídia, que disseminam informações que nem sempre condizem com as evidências científicas disponíveis. De acordo com Wannmacher ${ }^{7}$, é imprescindível haver absoluta ausência de conflito de interesses na tomada de decisão terapêutica (individual e coletiva) e, consequentemente, na prescrição de medicamentos.

Sabe-se que a indústria farmacêutica exerce uma influência muito forte na prescrição de medicamentos. A visita de propagandistas a consultórios, ambulatórios e hospitais constitui a ferramenta mais simples das usadas pela indústria farmacêutica para modificar padrões de prescrição e consumo de medicamentos. Segundo Wanmmacher ${ }^{7}$, para que o médico se mantenha atualizado em uma especialidade, é necessária a leitura de 19 artigos científicos por dia, de acordo com dados do National Institute for Health and Clinical Excellence (Nice) do Reino Unido. Devido à dificuldade de fazer frente à multiplicidade de novas informações, o médico, muitas vezes, se "atualiza" com as propagandas médicas. Usualmente, esse material de divulgação, patrocinado pelos fabricantes, não apresenta todas as informações exigidas para uma adequada indicação do produto ${ }^{7}$.

Na prática, ocorre certa pressão dos pacientes sobre o prescritor para receberem prescrição de fármacos dos quais tomaram conhecimento via propaganda direta em detrimento de outros de eficácia e segurança comprovadas. As "pílulas" são apresentadas como milagrosas, estimulando o desejo de usá-las para controle de problemas como sobrepeso, desatenção infantil, insônia, constipação, disfunção erétil, tabagismo, rugas de envelhecimento e manifestações da menopausa ${ }^{7}$.

Um estudo realizado por Trevisol ${ }^{8}$ mostra que, em uma escola de Medicina do Sul do Brasil, 85,4\% dos professores médicos participantes da pesquisa afirmaram que recebem ou já receberam visita de representantes da indústria farmacêutica, que, na maioria das vezes, deixam brindes (desde amostra grátis de medicamentos até viagens internacionais). Entre os professores médicos, 53,6\% consideraram que nunca ou raramente são influenciados pela indústria farmacêutica em sua 
prescrição e 53,7\% afirmaram acreditar que os colegas profissionais o sejam ${ }^{8}$.

Somando-se a isso, muitas prescrições geradas no serviço público de saúde brasileiro não apresentam os requisitos técnicos e legais, estabelecidos sobretudo pelas Leis $\mathrm{n}^{\circ} 5.991 / 73^{9} \mathrm{e}$ $\mathrm{n}^{\circ} 9.787 / 99^{10}$, o que pode comprometer a dispensação eficiente e a utilização correta dos medicamentos. Isto retroalimenta a demanda pelos serviços clínicos, muitas vezes em níveis mais complexos, diminuindo a relação custo/efetividade dos tratamentos, onerando de forma desnecessária os gastos com saúde e diminuindo a qualidade de vida dos pacientes. Além disso, as prescrições inadequadas ou mesmo ilegíveis, aliadas ao baixo nível socioeconômico-cultural dos pacientes brasileiros, são fatores relevantes na exposição das várias camadas que compõem a sociedade, em especial os idosos e as crianças, aos possíveis problemas relacionados com os medicamentos ${ }^{11}$.

O Uso Racional de Medicamentos foi definido na Conferência Mundial sobre Uso Racional de Medicamentos ocorrida em Nairobi, no Quênia, em 1985. Nesse momento, chegou-se ao consenso de que existe uso racional quando os pacientes recebem medicamentos apropriados as suas necessidades clínicas, em doses adequadas a suas particularidades individuais, por período de tempo necessário e com baixo custo para eles e para sua comunidade ${ }^{12}$.

O Uso Racional de Medicamentos envolve tanto o prescritor quanto o paciente (individual ou coletivo). Estes devem interagir de forma dinâmica. O prescritor tem papel determinante na conduta do paciente, mas este também, com suas expectativas e hábitos culturais que podem influenciar no uso dos medicamentos. A relação destes atores está permeada por uma série de questões que envolvem a capacidade de definição e implementação de uma política de medicamentos, as relações do mercado farmacêutico, particularmente no que se refere à propaganda médica, à organização da rede de serviços, em nível de educação da sociedade, a fatores culturais de modo geral e ao arcabouço legal ${ }^{3}$.

Neste processo, deve se enfatizar também o processo educativo dos usuários ou consumidores acerca dos riscos da automedicação, da interrupção e da troca de medicamentos prescritos por conta própria, bem como quanto à necessidade de acesso aos serviços médicos no tocante à dispensação e utilização dos medicamentos sujeitos a receita médica ${ }^{13}$.

Todos os profissionais da saúde devem estar comprometidos com políticas públicas que garantam a expansão da atenção à saúde, o que abrange as ações necessárias para a promoção do Uso Racional de Medicamentos. Este é o grande desafio proposto na atualidade, tanto para o governo, como também para as categorias profissionais e todos aqueles que podem contribuir, em seu dia a dia, para otimizar as ações que visam melhorar as condições de saúde e de vida da população brasileira $^{14}$.

Intervenções como a realização de cursos de farmacoterapia nos programas de estudos universitários, baseados em problemas concretos, a educação médica contínua como requisito para o bom desempenho dos profissionais e a educação da população a respeito dos medicamentos são consideradas essenciais para promover o uso racional de medicamentos ${ }^{4,15}$.

Este cenário demonstra que ações desenvolvidas para o ensino voltado à prescrição racional são essenciais para que a prática profissional se desenvolva em prol dos interesses da coletividade, em detrimento de interesses financeiros de determinados setores

\section{Ensino sobre o Uso Racional de Medicamentos: Construindo um Caminho}

De acordo com Lopes ${ }^{16}$, a introdução nas escolas médicas de métodos didáticos que estimulem o autoaprendizado deverá contribuir para a formação de profissionais capazes de selecionar adequadamente a fonte do conhecimento e de avaliar criteriosamente como transferir a informação para a prática médica. Além do mais, é importante que as escolas utilizem métodos didáticos que capacitem o estudante a pesquisar e criticar a informação científica, contribuindo, para desenvolver atitudes que resultem na melhoria da qualidade dos cuidados que o mesmo venha prestar a seus pacientes ao se tornar médico ${ }^{16}$.

De acordo com Hogerzeil et al. ${ }^{17}$, pesquisas realizadas no Canadá, Estados Unidos e Europa concluíram que o treinamento estruturado em farmacoterapia é relativamente infrequente. Em muitas escolas médicas, o ensino da farmacoterapia é caracterizado pela transferência de conhecimento sobre os medicamentos, mais do que pelo desenvolvimento de habilidade para lidar com os pacientes. Entretanto, desde a década de 1990, têm sido desenvolvidos programas educacionais com o objetivo de melhorar o ensino da farmacoterapia ${ }^{17}$.

A partir da experiência desenvolvida pela Universidade de Groningen, nos Países Baixos, a OMS vem incentivando a realização de cursos de formação sobre Uso Racional de Medicamentos, sendo que o primeiro curso a ser realizado na América Latina ocorreu na Universidad de La Plata, na Argentina $^{4,18}$.

No Brasil, o ensino sobre o Uso Racional de Medicamentos começou a ser propagado nas escolas de Medicina e demais cursos da área da saúde a partir de 2000. No total, até 2006, 458 profissionais da saúde e 309 estudantes universitários de 21 Estados brasileiros participaram de $\operatorname{cursos}^{18}$. Esta experiência 
é necessária no Brasil, onde não há Farmacoterapêutica como disciplina médica. A Farmacologia é ministrada no início dos cursos da área da saúde, como disciplina básica, sem inter-relação com a atividade profissional. Os cursos voltados ao ensino sobre uso racional de medicamentos constituem treinamento docente de caráter eminentemente prático, objetivando capacitar os participantes em metodologia de ensino direcionada à prescrição racional de medicamentos. A metodologia apresenta sequência lógica de passos dentro do processo de prescrever para resolver problemas de saúde de pacientes individualizados. A intenção, a partir de treinamento para docentes, é disseminar os princípios da prescrição racional de medicamentos entre estudantes das áreas da saúde - futuros prescritores e dispensadores —, intentando melhorar o nível da atenção à saúde nos países em desenvolvimento ${ }^{19}$.

A discussão sobre o ensino para o Uso Racional de Medicamentos é uma estratégia mundial, que no Brasil tem apoio da Organização Pan-Americana da Saúde (Opas), do Ministério da Saúde (MS) e da Agência Nacional de Vigilância Sanitária (Anvisa), em parceria com as principais universidades e centros de ensino e pesquisa do País. Por isso, o ensino para o Uso Racional de Medicamentos ganhou espaço e respeito em muitas universidades, entre docentes, discentes e profissionais da área da saúde. De acordo com Wanmmacher ${ }^{19}$, além do ensino voltado à prescrição racional, outras estratégias para promover o Uso Racional de Medicamentos são a priorização de medicamentos essenciais e o estabelecimento de condutas pautadas pelas melhores evidências contemporâneas disponíveis.

O método utilizado nos cursos que propagam o ensino sobre o Uso Racional de Medicamentos utiliza a farmacoterapia baseada em problemas, em que o aluno aprende a buscar e analisar as evidências disponíveis e a tomar a decisão clínica. Este aspecto é central para o treinamento das habilidades cognitivas, que requer o treinamento baseado em problemas em pequenos grupos de discussão. Adicionalmente, são necessários métodos educacionais específicos para ensinar habilidades de comunicação, tais como o uso de pacientes simulados e o ensino à beira do leito ${ }^{17}$.

De acordo com o modelo normativo proposto pela OMS para o ensino da farmacoterapia racional, num primeiro momento os estudantes são levados ou conduzidos a selecionar tratamentos padronizados para as doenças comuns, resultando em um conjunto de medicamentos de primeira escolha, chamados de medicamentos-I (individuais). No curso do desenvolvimento de seus medicamentos-I, os estudantes são ensinados a consultar protocolos de tratamento nacionais e internacionais, formulários, livros-texto e outras fontes de informação sobre medicamentos. Em seguida, eles são ensinados a aplicar o conjunto de medicamentos-I a problemas específicos de pacientes, usando um esquema de resolução de problemas composto por seis passos: (1) definir o problema do paciente; (2) especificar o objetivo terapêutico; (3) verificar a conveniência de seus medicamentos-I e escolher o tratamento para esse paciente individual; (4) escrever a prescrição; (5) informar e instruir o paciente; (6) monitorar e/ou interromper o tratamento ${ }^{17,20}$.

Conforme enfatizam Hogerzeil et al. ${ }^{17}$, com a racionalidade subjacente a essa abordagem é que o estudante desenvolve, em algum momento de seus estudos ou precocemente em sua carreira, a definição de um conjunto de medicamentos que usará regularmente a partir de então.

\section{A Experiência no Curso de Medicina da Unesc: Resultados e Discussão}

A Unesc oferece um curso de graduação em Medicina com uma proposta inovadora, que objetiva responder às exigências e aos desafios contemporâneos, considerando o ensino médico como um processo contínuo, que se inicia na graduação e continua na vida profissional. Busca um ensino voltado às necessidades regionais na qual ela se insere, mas preparando o egresso para enfrentar e superar desafios de outras realidades. Estimula o acadêmico a ser um profissional criativo, isto é, um profissional que saiba reorganizar o conhecimento, construindo novos caminhos, encontrando novas saídas para os problemas do dia a dia ${ }^{21}$.

A concepção didático-pedagógica que utiliza metodologias ativas de ensino-aprendizagem requer transformações fundamentais na organização e desenvolvimento da prática pedagógica, como no caso da Aprendizagem Baseada em Problemas. De acordo com Silva e Delizoicov ${ }^{22}$, esta metodologia parte do pressuposto da centralidade e da autonomia do aluno no processo de aprendizagem. Apresenta como principais características a integração de conteúdos disciplinares estruturados sempre no contexto de um problema orientado para a discussão e resolução em grupo.

Sob essa perspectiva, foi concebido um projeto de pesquisa e extensão na Unesc, dentro do Programa de Pesquisa e Ação Comunitária 2 ${ }^{a}$ Edição (PPAC II), que foi aprovado e recebeu apoio e financiamento da Diretoria de Pesquisa e da Diretoria de Extensão e Ação Comunitária. No PPAC II, a Unesc fomenta projetos que tenham real inserção na realidade social e regional sul-catarinense. $\mathrm{O}$ projeto aprovado intitulou-se “Promoção do Uso Racional de Medicamentos para a comunidade dos bairros Pinheirinho, Paraíso, Tereza Cristina, Santa Augusta e Grande Santa Luzia", tendo duas vertentes de 
atuação: a primeira visou capacitar alunos dos cursos de Medicina e de Farmácia da Unesc na lógica do Uso Racional de Medicamentos por meio da Aprendizagem Baseada em Problemas, conforme o modelo normativo proposto pela OMS ${ }^{17,20}$. Já a segunda vertente objetivou desenvolver ações educativas na comunidade dos bairros supracitados, envolvendo temas como Uso Racional de Medicamentos, promoção da saúde e prevenção de doenças. O projeto teve início em março de 2006 e término em fevereiro de 2008.

A metodologia utilizada na primeira vertente deste projeto foi estruturalmente semelhante àquela proposta pela OMS, geralmente composta por dois momentos: as plenárias, onde foram abordados de forma teórica os temas de trabalho, e, em seguida, o estudo em grupos tutoriais. O projeto contou com cinco acadêmicos bolsistas (quatro do curso de Medicina e uma do curso de Farmácia), os quais foram capacitados, servindo como multiplicadores para os demais alunos posteriormente.

A seguir serão apresentadas, sucintamente, as etapas realizadas na capacitação. A primeira etapa objetivou que os alunos aprendessem a buscar e selecionar informações científicas para responder perguntas clínicas, em conformidade com os critérios da Medicina Baseada em Evidências. Nesse momento, foram abordados, em plenárias, assuntos como a busca, seleção e análise de fontes de informação científica; delineamento de pesquisas clínicas e leitura crítica de artigos científicos, listas de medicamentos essenciais e Programas de Medicamentos disponibilizados pelo Sistema Único de Saúde (SUS). Os temas abordados inicialmente foram fundamentais para que os alunos desenvolvessem um raciocínio terapêutico racional de acordo com a realidade do sistema de saúde.

É essencial que os alunos saibam distinguir fontes fidedignas, éticas e isentas, o que exclui qualquer informação proveniente do produtor de medicamentos, que tem interesses comerciais no produto, muitas vezes suplantando o dever de informar correta e cientificamente. Mesmo artigos em revistas especializadas, consensos, diretrizes nacionais e internacionais podem estar permeados por interesses comerciais ${ }^{23}$. Além disso, foi abordada a questão do acesso aos medicamentos no SUS, que ocorre por meio de programas governamentais. Em contraponto ao fornecimento de medicamentos por intermédio dos programas vigentes, tem ocorrido uma demanda crescente mediante processos judiciais, em que os magistrados obrigam o Estado a adquirir medicamentos, essenciais ou não, para atender as necessidades de determinado paciente, em detrimento da coletividade ${ }^{24}$. É importante ressaltar que isso vai de encontro às políticas públicas adotadas no País, gerando um aumento nos gastos com saúde de forma irracional. Este fato é de suma importância e precisa ser conhecido e compreendido tanto por estudantes quanto por profissionais, sejam médicos ou farmacêuticos, devendo, por isso, ser discutido nos cursos de graduação.

Na segunda etapa, os estudantes seguiram o modelo normativo para a farmacoterapia racional, estruturado pela OMS, descrita no Guia para a Boa Prescrição Médica. Portanto, nos grupos tutoriais, os estudantes foram conduzidos a selecionar tratamento padronizado para hipertensão arterial sistêmica, resultando em um conjunto de medicamentos de primeira escolha, chamados de medicamentos-I, conforme descrito anteriormente ${ }^{17,20}$

$\mathrm{Na}$ terceira etapa do projeto, os cinco acadêmicos bolsistas capacitados passaram a executar o papel de multiplicadores, realizando este mesmo treinamento para mais 35 alunos ( 25 do curso de Medicina e 10 do curso de Farmácia), desde iniciantes no curso até alunos do Internato Médico. Todos passaram pelo mesmo processo citado. A utilização da multiplicação por meio de recém-formados no ensino sobre o Uso Racional de Medicamentos é fato corrente em todos os cursos realizados no Brasil, nos quais um indivíduo que participou de um curso passa a ser tutor de um grupo de alunos em um curso posterior. Isto solidifica o conhecimento adquirido anteriormente e promove uma propagação muito mais rápida do assunto.

Finalmente, na segunda vertente de atuação do projeto, todos os acadêmicos envolvidos na capacitação sobre o Uso Racional de Medicamentos passaram a desenvolver outras atividades de cunho educativo para a comunidade, visando divulgar o Uso Racional de Medicamentos, entre outros assuntos relacionados à promoção da saúde, conforme segue: (a) elaboração de folder e banner, utilizados nas ações educativas com a comunidade, sobre os seguintes assuntos: cuidados no armazenamento de medicamentos, prevenção e cuidados na hipertensão arterial sistêmica, prevenção e cuidados no diabetes mellitus; (b) realização de cinco palestras para grupos de hipertensos e diabéticos das Unidades Básicas de Saúde dos bairros abrangidos pelo projeto, totalizando cem ouvintes; (c) realização de 12 palestras em escolas da rede pública municipal de Criciúma (SC) sobre temas diversos, como sexualidade e métodos contraceptivos, com participação de 490 alunos; (d) realização de cinco palestras sobre temas da atividade de farmacologia, como tratamento de dislipidemias, uso de anti-inflamatórios e analgésicos para os alunos do curso de Medicina da Unesc, totalizando 85 ouvintes; (e) realização de uma palestra sobre os problemas acarretados pelo uso de drogas para internos do Presídio do Bairro Santa Augusta (Criciúma/ $\mathrm{SC})$, contando com a presença de nove ouvintes; (f) capacitação das agentes comunitárias de saúde dos bairros Paraíso e 
Tereza Cristina, de Criciúma (SC), sobre armazenamento de medicamentos, com cinco participantes; (g) participação em congressos e fóruns locais, por meio de exposição de pôster, com o objetivo de difundir a proposta deste projeto.

De maneira geral, foram alvo das ações realizadas a partir deste trabalho 724 pessoas, entre acadêmicos da Unesc e cidadãos da comunidade de Criciúma (SC), com medidas consideradas simples e de baixo custo.

A avaliação dos resultados gerados a partir deste trabalho foi realizada de forma subjetiva, a partir do relato dos próprios participantes. De acordo com a percepção dos acadêmicos, os resultados deste treinamento se fazem presentes na vida profissional destes, muitos já formados. Ao atuarem no mercado de trabalho, diariamente estes profissionais se deparam com a necessidade de escolha de tratamentos, em que se leva em consideração não só a doença que o indivíduo apresenta, mas também o contexto econômico-social no qual o paciente se insere. O médico capacitado no ensino sobre Uso Racional de Medicamentos desenvolve uma visão de racionalização da terapêutica baseada na busca de conhecimentos científicos confiáveis que embasem determinada conduta. Sem esta capacitação, a atitude destes novos profissionais seria a mesma que vem ocorrendo ao longo dos anos, ou seja, o médico sai da faculdade e repete no dia a dia as "experiências" de seus preceptores, sem elaborar um raciocínio clínico para determinado caso.

A Unesc e a Direção dos cursos de Medicina e Farmácia apoiaram a iniciativa deste trabalho e colaboraram abertamente para sua execução, tanto nos aspectos táticos quanto nos operacionais. Entretanto, durante a execução do projeto, surgiram algumas barreiras. Uma delas referiu-se à dificuldade de captar mais acadêmicos para participar das capacitações, resultando em número relativamente reduzido de alunos na etapa de multiplicação. Muitos acadêmicos alegaram falta de tempo, já que estavam constantemente atarefados com atividades de ensino e pesquisa obrigatórias relacionadas ao curso, e esta atividade se caracterizava como não obrigatória.

Outra dificuldade ocorreu durante as ações na comunidade, já que algumas vezes os alunos se deslocavam até a Unidade Básica de Saúde e não encontravam público para realizar as palestras e prestar orientações durante as reuniões dos grupos de hipertensos e diabéticos. Além disso, outra barreira percebida se refere à resistência de alguns prescritores em concordar e/ou se adequar aos moldes da prescrição racional de medicamentos, visto que os alunos que participavam da capacitação partiam para os estágios em serviço com uma visão mais crítica com relação à prescrição. Eventualmente, acompanhavam algumas condutas terapêuticas contraditórias em relação à seleção de medicamentos baseada nas melhores evidências disponíveis.

Com relação à mescla de acadêmicos de cursos diferentes nas atividades do projeto, a avaliação posterior feita com os próprios acadêmicos demonstrou que isto enriqueceu muito as discussões nos grupos de trabalho, pois as áreas se inter-relacionam e se complementam. Considera-se essencial que os grupos de trabalho sejam constituídos por alunos de dois ou mais cursos da área da saúde, podendo incluir, além da Medicina e Farmácia, a Odontologia e a Enfermagem, para obter pluralidade de ideias, fomentar a discussão e reforçar a necessidade de trabalho em equipe. Além disso, a convivência com acadêmicos de outras áreas, ainda na fase de formação universitária, proporciona ao aluno adquirir a noção do campo de saberes do outro profissional e, ainda, iniciar a criação do vínculo entre as profissões, essencial para o trabalho em equipes multidisciplinares e interdisciplinares.

\section{CONCLUSÕES}

No desenvolver deste projeto, ficou evidente a pertinência da metodologia utilizada e o despertar motivacional dos participantes. A maioria dos alunos que concluíram a capacitação ficou sensibilizada pelo tema Uso Racional de Medicamentos, demonstrando seu interesse e uma nova visão com relação ao assunto.

A abordagem de incentivo ao estudo sobre Uso Racional de Medicamentos fornece ao acadêmico uma competência que é pouco enfatizada no ensino médico tradicional, já que se apropria de critérios de racionalidade para a tomada de decisão em saúde. Geralmente, a forma como esse aprendizado ocorre é baseada na repetição das experiências prévias, adquiridas principalmente nas residências médicas.

Os participantes da capacitação sobre Uso Racional de Medicamentos acreditam que esta abordagem deveria ser incorporada às atividades de Farmacologia de todos os cursos de Medicina, pois se trata de uma abordagem de ensino diferenciada, que estimula o aluno a aprender a buscar informações e a tomar decisões próprias, a partir do seu raciocínio clínico. Através dela, é possível que os alunos tenham uma visão mais abrangente do que é verdadeiramente uma prescrição médica, da racionalidade envolvida, bem como dos seus riscos e benefícios.

Os acadêmicos envolvidos neste trabalho encontraram nele a possibilidade de estabelecer relações mais próximas com a comunidade, observando os pontos cruciais em relação ao uso de medicamentos e ao seu armazenamento, que necessita de aconselhamento. $\mathrm{O}$ contato com os pacientes fora do consultório, com as agentes comunitárias de saúde e com os 
profissionais da área de enfermagem proporcionou uma nova visão em relação ao uso de medicamentos e suas principais falhas, apontando as atitudes de relevância social que devem ser tomadas pelos mesmos na multiplicação do conhecimento adquirido na universidade a partir deste trabalho.

Pretende-se, a partir deste trabalho, incentivar novos acadêmicos da área da saúde da Unesc a dar continuidade às intervenções já realizadas na comunidade, bem como a formar um grupo permanente de estudos em Uso Racional de Medicamentos.

\section{REFERÊNCIAS}

1. Fuchs FD, Wannmacher L. Conduta Terapêutica Embasada em Evidências. Rev Ass Med [periódico na internet]. 2000. 46(3) [acesso em 17 out. 2008]; 237-241. Disponível em: www.scielo.br/pdf/ramb/v46n3/3083.pdf

2. Sackett DL, Straus SE, Richardson WS, Rosenberg W, Haynes RB. Medicina Baseada em Evidências: prática e ensino. $2^{a}$ ed. Porto Alegre: Artmed; 2003.

3. Marin N, Luiza VL, Osório-de-Castro CGS, Machado-dos-Santos S (orgs.). Assistência farmacêutica para gerentes municipais. Rio de Janeiro: OPAS/OMS; 2003.

4. Organizacion Mundial de la Salud. Promoción del Uso Racional de Medicamentos: componentes centrales. Perspectivas políticas sobre medicamentos de La OMS. 2002 sept.; 5:1-6.

5. Beria JU. Prescrição de Medicamentos. In: Duncan BB, Schmidt MI, Giugliani ERJ. Medicina Ambulatorial: condutas de atenção primária baseadas em evidências. $3^{\mathfrak{a}}$ ed. Porto Alegre: Artmed, 2004. p.143-9.

6. Pepe VLE, Castro CGSO. A interação entre prescritores, dispensadores e pacientes: informação compartilhada como possível benefício terapêutico. Cad Saúde Pública [periódico na internet]. 2000 [acesso em 17 jul. 2009];16(3):815-22. Disponível em: http:/ / www.scielo.br/ pdf/csp/v16n3/2966.pdf.

7. Wannmacher L. A ética do medicamento: múltiplos cenários. Uso Racional de Medicamentos: temas selecionados [periódico na internet]. Jul. 2007 [acesso em 06 dez. 2008];4(8):01-06. Disponível em: portal.saude.gov.br/portal/arquivos/pdf/v4n8_etica_medicamentos.pdf

8. Trevisol DJ. Influência da propaganda da indústria farmacêutica na prescrição de medicamentos em Escolas de Medicina: a experiência em Tubarão, Santa Catarina. Tubarão; 2006. Mestrado [Dissertação] — Universidade do Sul de Santa Catarina.

9. Brasil. Lei no 5.991 de 17 de dezembro de 1973. Dispõe sobre o controle sanitário do comércio de drogas, medicamentos, insumos farmacêuticos e correlatos, e da outras providências [on line]. Diário Oficial da União. Brasília, 19 dez. 1973. [acesso em 19 fev. 2002]. Disponível em: http:/ /e-legis.anvisa.gov.br/leisref/public/showAct.php.

10. Brasil. Lei no 9.787 de 10 de fevereiro de 1999. Altera a Lei $\mathrm{n}^{\mathrm{o}}$ 6.360, de 23 de setembro de 1976, que dispõe sobre a vigilância sanitária estabelece o medicamento genérico, dispõe sobre a utilização de nomes genéricos em produtos farmacêuticos e dá outras providências. Diário Oficial da União. Brasília, 10 fev. 1999. [acesso em 19 fev. 2002]. Disponível em: http://e-legis.anvisa.gov.br/leisref/public/ showAct.php?id=16622\&word.

11. Lyra Junior DP, Prado MCTA, Abriata JP, Pelá IR. As Prescrições Médicas como Causadoras de Risco para Problemas Relacionados com os Medicamentos. Seguim Farmacoter. 2004;2(2)86-96.

12. World Health Organization. The Rational use of drugs: report of the conference of experts. Nairóbi, 25-29 november 1985. Geneva: WHO; 1987.

13. Brasil. Ministério da Saúde. Secretaria de Políticas de Saúde. Departamento de Atenção Básica. Política Nacional de Medicamentos, 2001 [on line]. Brasília: Ministério da Saúde, 2001. (Série C. Projetos, Programas e Relatórios, n.25). [acesso em 15 abr. 2003] Disponível em: www.opas.org.br / medicamentos/site/UploadArq/pnm.pdf.

14. Bermudez J. Medicamentos: uma questão polêmica. Rev Ciência Hoje. 2000;27(161).

15. Barros JAC. Políticas farmacêuticas: a serviço dos interesses da saúde? Brasília: UNESCO; 2004.

16. Lopes AA. Medicina Baseada em Evidências: a arte de aplicar o conhecimento científico na prática clínica. Rev Assoc Med Bras [periódico na internet]. 2000 [acesso em 17 jul. 2009]; 46(3): 285-8. Disponível em: http:/ / www.scielo. $\mathrm{br} / \mathrm{pdf} / \mathrm{ramb} / \mathrm{v} 46 \mathrm{n} 3 / 3089 . \mathrm{pdf}$.

17. Hogerzeil HV, Barnes KI, Henning RH, Kocabasoglu YE, Möller H, Smith AJ, et al. Guia do Instrutor em Práticas da Boa Prescrição Médica. Genebra: Organização Mundial de Saúde; 2001.

18. Fitzgerald J. Estratégias para o Uso Racional de Medicamentos. [Trabalho apresentado no $2^{\circ}$ Congresso Brasileiro Sobre Uso Racional de Medicamentos; 15 - 18 out. 2007; Florianópolis, SC].

19. Wannmacher L. Uso Racional de Medicamentos: perspectivas nacionais [on line]. Trabalho apresentado no Seminário sobre Uso Racional de Medicamentos;. abr. 2006 [acesso em 13 out 2008]; Curitiba, PR. Disponível em: http: / / 200.189.113.52/ftp/Visa/farmaco/URM_LenitaWannmacher.pps. 
20. Vries TPGM, Henning RH, Hogerzeil HV, Fresle DA. Guia para a Boa Prescrição Médica. Organização Mundial da Saúde: Artmed. Porto Alegre; 1988.

21. Pires MMS, Pereima MJL, Miranda SM, Nassar SM, Cechinel C, Peck GMF, et al. Aprendizagem Baseada em Problemas: aprendendo a conhecer um currículo inovador. Criciúma: Unesc; 2003.

22. Silva WB, Delizoicov D. Problemas e problematizações: implicações para o ensino dos profissionais da saúde. Ensino Saúde e Ambiente [periódico na internet]. 2008 [acesso em 05 fev. 2009];1(2):14-28. Disponível em: www.unipli. com.br/mestrado/.../Texto\%202\%20Delizoicov.pdf

23. Wannmacher L. Quanto é evidente a evidência na saúde? Uso Racional de Medicamentos: temas selecionados [periódico na internet]. 2006. [acesso em 06 dez. 2008];3(5):0106. Disponível em: http:/ / www.opas.org.br/medicamentos/site/UploadArq/HSE_URM_EME-0305.pdf.

24. Pereira JR. Análise das demandas judiciais solicitando medicamentos encaminhados à Diretoria de Assistência Farmacêutica da Secretaria de Estado da Saúde de Santa Catarina, nos anos de 2003 e 2004. Florianópolis; 2006. Mestrado [Dissertação] — Universidade Federal de Santa Catarina.

A autora, Iane Franceschet de Sousa, esteve contratada como docente na Universidade do Extremo Sul Catarinense (Unesc), Criciúma/SC, até agosto de 2007, período no qual foi desenvolvido o trabalho de que trata este artigo.

\section{CONTRIBUIÇÃO DOS AUTORES}

Iane Franceschet de Sousa contribuiu na elaboração do projeto, redação e revisão final do artigo, desde a introdução, revisão da literatura, relato da atividade prática, resultados e discussão, bem como nas conclusões. Andressa Biscaro contribuiu na elaboração do projeto, revisão da literatura, relato da atividade prática, resultados e discussão, conclusões e redação final. Fernanda Biscaro contribuiu na elaboração do projeto, revisão da literatura e redação final. Marcelo Soares Fernandes contribuiu na elaboração do projeto, revisão da literatura e redação final.

\section{CONFLITO DE INTERESSES}

Declarou não haver.

\section{ENDEREÇO PARA CORRESPONDÊNCIA}

Iane Franceschet de Sousa

Rua Udinese, 201 casa 4 Residencial Villas de Astúria III. Bairro Planalto - Campo Grande

CEP. 79009-705 MS

E-mail: ianefran@gmail.com 